\title{
Cold spraying: A low temperature variant of thermal spray techniques to deposit metallic materials
}

\author{
S. Kumar a,", Naveen M Chavan a, D. Srinivasa Rao a \\ a Centre for Engineered Coatings, International Advanced Research Centre for Powder Metallurgy and New Materials \\ (ARCI), Balapur Po., Hyderabad 500 005, India.
}

*Corresponding Author skumar@arci.res.in

(S. Kumar)

Received : $10^{\text {th }}$ February 2019 Accepted : $31^{\text {st }}$ March 2019

\begin{abstract}
Cold spraying is a novel material deposition process in which micron size particles are accelerated to supersonic velocity on to a metallic substrate to obtain thick and dense coatings. Unlike other thermal spray coatings, the bonding mechanism is completely different. In conventional thermal spray techniques, melting and solidification upon impact dominates the bonding mechanism. In cold spraying, Plastic deformation induced adiabatic shear instability governs the bonding process in which adiabatic temperature rise, plastic strain at interface and flow stress collapse play a crucial role. Variety of material including pure metals, alloys, composites and cermets have been deposited using cold spraying for variety of applications. In this article, a brief introduction about the bonding mechanism and potential applications of cold spraying is being discussed.
\end{abstract}

Keywords: Cold spray, bonding mechanism

\section{Introduction}

In the last decade, cold spraying has emerged as a novel coating technique for deposition of high density metallic coatings used for variety of applications. This process involves deposition at relatively very low temperature and high pressure compared to other thermal spray techniques thus avoids adverse effects such as oxidation and phase transformation. In cold spraying micron size metallic or alloy powders are accelerated through De Laval type converging - diverging nozzle to supersonic velocity by compressed Air/Nitrogen/Helium. The accelerated high velocity metallic particles impacts on to the metallic substrate to obtain thick high dense coatings. In general, the degree of plastic deformation determines the inter splat bonding in the coating. The bonding mechanism is unique in the cold spray process as this is the only technique among all thermal spray variants in which the feedstock is in fully solid state at the moment of impact with the substrate or already deposited layers without any oxidation and / or phase transformation.

\section{Bonding mechanism}

Upon impact, both feedstock and substrate undergoes severe plastic deformation. The bonding time is typically few nano seconds. The bonding mechanism is generally governed by the plastic deformation induced adiabatic temperature rise, exponential increase in plastic strain and collapse of flow stress to near zero at impacting interfaces. This phenomena is called as adiabatic shear instability $[1,2]$. In other words, the initial kinetic energy of the impacting particle is dissipated into plastic dissipation energy. A small portion of the elastic strain energy is also inevitable which adversely influences the bonding process. In general, the ratio between the plastic deformation energy and elastic strain energy gives an estimation of bonding. The interface temperature generally goes to the near melting temperature of impacting bodies upon impact. In other words, during the impact, the material undergoes strain and strain rate hardening and the combined effect competes with thermal softening effect. When the thermal softening dominates over hardening due to strain and strain rate, bonding occurs. The velocity of the impacting particle decides the quantum of thermal softening and strain and strain rate hardening. Critical velocity is a velocity at which thermal softening dominated over stain hardening thus generates bonding upon impact. For a successful cold spray deposition, feedstock must be accelerated above its critical velocity.

\section{Understanding bonding mechanism}

It is very difficult to understand the bonding process since the bonding is highly localised. High resolution microscopy methods such as Focussed Ion Beam (FIB) assisted Transmission Electron Microscopy (TEM) techniques are being used to understand the bonding states. Owing to the computational simulation facility, the bonding process in cold spraying can be easily predicted by high speed computing. For this purpose, Johnson - Cook constitutive 

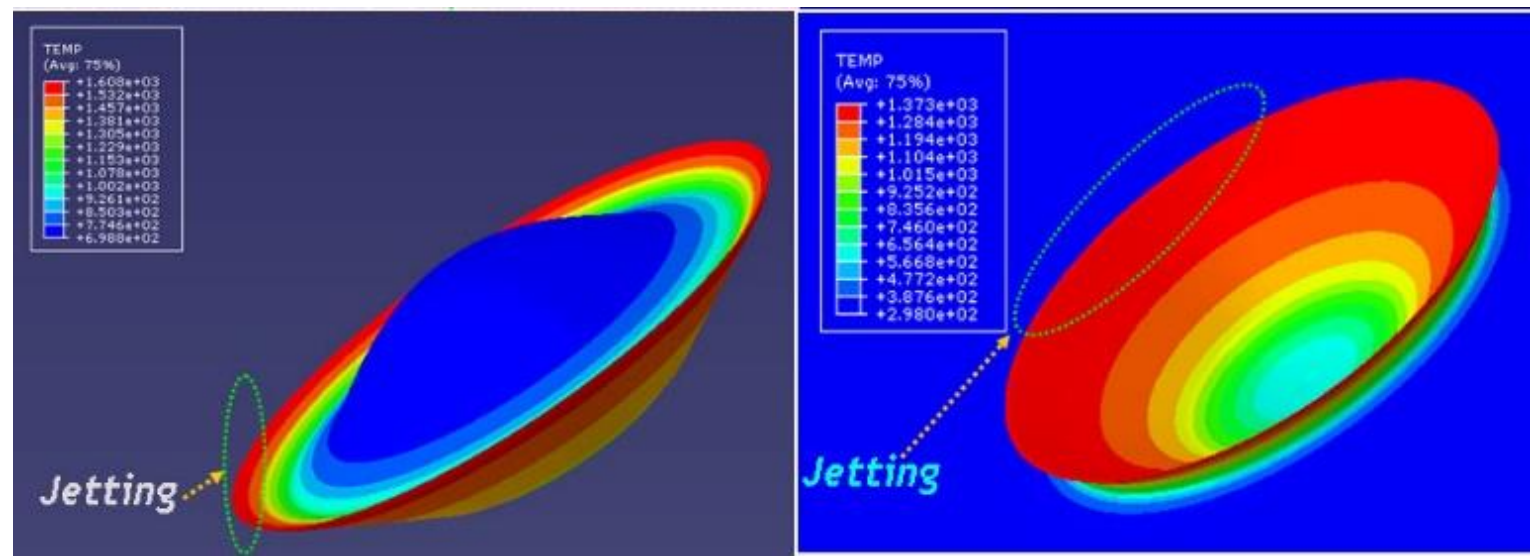

Fig.1. Deformed Splat (a) and Crater (b) of copper

Equation [3] which governs the metal plasticity can be applied which is shown as below,

$$
\sigma=\left[A+B \varepsilon_{p}^{n}\right]\left[1+C \ln \left(\frac{\dot{\varepsilon}_{p}}{\dot{\varepsilon}_{n}}\right)\right]\left[1-\left(T^{*}\right)^{m}\right]
$$

This equation takes care of strain hardening, strain rate hardening and thermal softening of the impacting impacts during deformation. The high strain rate materials data used in the equation may be derived from extreme experiments such as split Hopkinson bar test. Fig. 1a and Fig. $1 b$ show the deformed particle and substrates respectively. The jetted portion undergoes the adiabatic shear instability process.

\section{Cold spraying process}

Compressed gas is used to accelerate the powder particles through de Laval nozzle. For this very purpose, the process gas is slightly preheated for the purpose of expansion. Stagnation pressure and stagnation temperature of the gas are two primary variables to obtain cold spray coatings. Apart from these, other variable such as powder size, density, standoff, design of nozzle, spray angle, powder - substrate combination often plays a crucial role to obtain optimized coating for possible applications. Accelerated powder deforms and forms a splat and all splats together form dense pore free thick metallic coatings. Fig. $2 a$ and Fig. $2 b$ shows the copper splat and coating cross section respectively. One can show the deformed splat with jetted portion at the periphery and flattened splats in the coatings.

\section{Applications of cold spray coatings}

A wide variety of materials such as metals, alloys, composites and cermets have already been deposited using cold spray technique [4 7]. Aerospace, automotive, defence and other sectors are actively involved in adopting cold spray technique as one among their important processing techniques. Since cold spraying is one among the high rate deposition process, this technique can be employed into additive manufacturing, rapid prototyping etc.

A considerable attention is being given to repairing industrial components for enhancing their life. Apart from the above, traditional overlay coatings for functional applications such as electrical conductive coatings, corrosion resistance coatings are being developed using cold spray techniques. Presently many industries and researchers globally are under perusal to develop neomaterial coatings such as bulk metallic glasses, High entropy alloys, functional metal matrix composites and high temperature carbides for possible industrial and strategic applications.

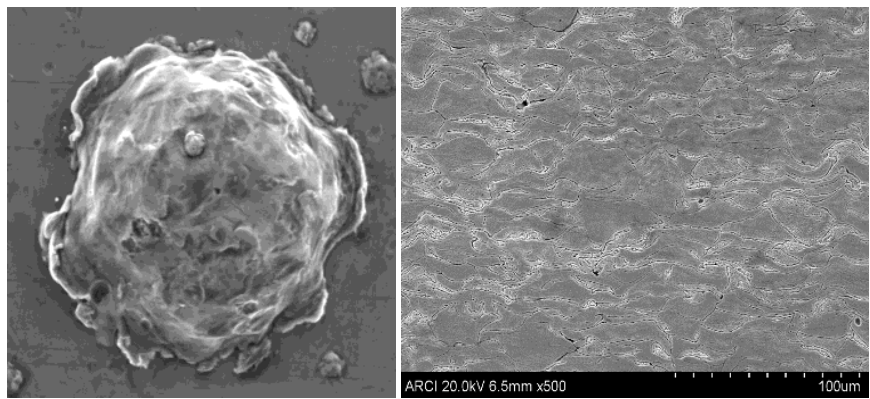

Fig.2. SEM images of copper (a) splat and (b) coating
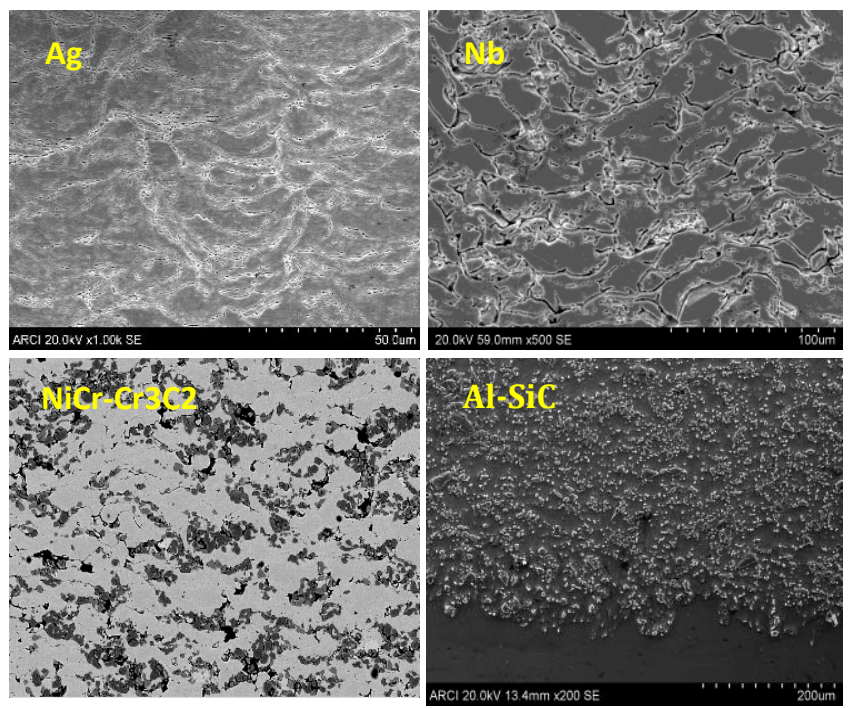

Fig.3 SEM microstructures of cold sprayed (a) Ag, (b) $\mathrm{Nb}$, (c) $\mathrm{NiCr} \sim \mathrm{Cr} 3 \mathrm{C} 2$ and (d) $\mathrm{Al} \sim \mathrm{SiC}$ 


\section{Reference}

[1] H. Assadi, F. Gartner, T. Stoltenhoff and H. Kreye, Bonding mechanism in cold gas spraying, Acta Mater. 51 (2003) 4379 4394.

[2] G. Bae, Y. Xiong, S. Kumar, K. Kang and C. Lee, General aspects of interface bonding in kinetic sprayed coatings, Acta Mater. 56 (2008) 4858 4868.

[3] S. Kumar, G. Bae, K. Kang, S. Yoon and C. Lee, Effect of powder state on the deposition behaviour and coating development in kinetic spray process, J. Phys. D: Appl. Phy. 42 (2009) $075305 \sim 8$.

[4] S. Kumar, V. Vidyasagar, A. Jyothirmayee and S. V. Joshi, Effect of heat treatment on mechanical properties and corrosion performance of cold sprayed tantalum coatings, J. Therm. Spray. Technol. 25 (4) (2016) 745 756.

[5] S.Kumar, A.Jyothirmayi, Nitin Wasekar and S.V. Joshi, Influence of annealing on mechanical and electrochemical properties of cold sprayed niobium coatings, Surf.Coat Technol, 296 (2016),124 135.

[6] Naveen Manhar Chavan, M. Ramakrishna, P. Sudarshan Phani, D. Srinivasa Rao and G. Sundararajan, The influence of process parameters and heat treatment on the properties of cold sprayed silver coatings, Surf. Coat. technol, 205(2011) 4798 4807.

[7] Naveen Manhar Chavan, B. Kiran, A. Jyothirmayi, P. Sudarshan Phani and G. Sundararajan, The Corrosion Behavior of Cold Sprayed Zinc Coatings on Mild Steel Substrate, J Therm Spray Technol, 22(2013)463 470.

\section{About The License}

Copyright by Sri Shakthi Institute of Engineering and Technology. All rights reserved. This article is licensed under a Creative Commons Attribution 4.0 International License. https://creativecommons.org/licenses/by/4.0/ This article may not be reproduced in any form or by any means, electronic or mechanical, including photocopying, recording or any information storage and retrivel system now known or to be invented, without written permission form the Copyright owner. 